\title{
Upper gi endoscopy in relation with carcinoma stomach
}

\author{
Dr. Pradeep Ghimire FRCS Ed. MS ${ }^{1}$ \\ ${ }^{\text {I}(C o n s u l t a n t ~ S u r g e o n, ~ F i s h t a i l ~ H o s p i t a l ~ a n d ~ R e s e a r c h ~ C e n t r e, ~ G a i r a p a t a n, ~ P o k h a r a, ~ N e p a l) ~}$
}

\begin{abstract}
Objectives: To find out Incidence of carcinoma stomach in patients who underwent upper GI endoscopy To identify different pathological conditions in patients who underwent upper GI endoscopy

Materials \& Methods: A Prospective study was done in seven hundred sixty patients in a period of one and half years in Fishtail Hospital, Gairapatan, and Pokhara, Nepal from April 2008 to September 2010. Findings were analyzed in different forms considering their age, sex, site, and their specific pathology with Microsoft Excel 2007. Most of endoscopies were done as out-patient basis (98\%). Local anesthetic (4\% xylocaine gargle) was used before the procedure.

Results: This study shows 58\% females (M: F-1:1.38) and of 25-45 age groups are mostly affected from Upper G. I. problems. Commonest finding was gastroduodenitis (28.9\%) and in $1.57 \%$ carcinoma stomach was detected.

Conclusion: Fiber-optic endoscopy is a safe and commonly used investigation. It is important that all personnel under taking this procedure are adequately trained and that resuscitation facilities are always available.
\end{abstract}

Keywords —carcinoma stomach, ulcer, upper gastrointestinal endoscopy

\section{INTRODUCTION}

The human gut is long and tortuous. Diagnosis and localization of its afflictions has relied for many decades on barium radiology which provides indirect data in black and white. Then rigid open-ended endoscopes have long allowed direct visual examination of the proximal $40 \mathrm{~cm}$ and distal $25 \mathrm{~cm}$ of the gut. Semiflexible lens gastroscopes were introduced in the 1930 and 1940s. With the introduction of fibreoptics since late 1960 s, the diagnostic situation has changed dramatically.

Flexible endoscopy is more sensitive than conventional radiology in the assessment of the majority of gastro duodenal conditions. Gastrointestinal fibrescopes are not only used for diagnosis, it is used in many occasion for different therapeutic purposes like: passing ,feeding or suction tubes, removal of foreign bodies, snare of polyps, sphincterotomy and removal of CBD stones, dilatation of esophageal strictures, diathermy, sclerotherapy \& laser photocoagulation.

Although the morbidity and mortality associated with upper G. I. endoscopy is extremely low (serious 1 in $500 \&$ death 1 in 10,000 procedures), it is not without hazard. We may encounter with medication reactions, aspiration pneumonia, perforations, instrumental impaction, cardiac dysrrhythmias \& transmission of infections. During endoscopy we may get patient distress in the form of choking, pain excessive air in sufflation disorientation, congenital malrotation, fluid or food, complex surgical procedure, regurgitation \& aspiration.

\section{MATERIALS AND METHODS}

A Prospective study was done in seven hundred sixty patients in a period of one and half years in Fishtail Hospital, Gairapatan, Pokhara from April 2008 to September 2010. Findings were analysed in different forms considering their age, sex, site, and their specific pathology with Microsoft Excel 2007. Most of endoscopies were done as out-patient basis (98\%). Local anesthetic (4\% xylocaine gargle) was used before the procedure. Fiber optic Fusinon Video-endoscope was used for the procedure.

Patients coming to this hospital directly \& referred from other clinicians in the period of one and half years were included in this study. Seven hundred sixty patients had endoscopic examinations after their formal consent. Indications for examinations were dyspepsia after 40 years of age, no improvement after two weeks of medical treatment, features of gastric outlet obstruction, haematemesis or malena, dysphasia. 
Procedure was performed early in the morning with premedication of local anesthetic (4\% xylocaine) gargle. No sedation was given. Except in two cases, there was no difficulty experienced (one has growth in esophagus \&bleeding \& other was so afraid).

During the procedure if the lesions were suspicious, proper biopsy were taken and sent histopathological examinations, mostly in cases of gastric ulcers. In cases of duodenal ulcers, biopsy was used to identify Helicobacter pylori infection.

Fiberoptic Fusinon Video-endoscope was used for the procedure.

\section{RESULTS}

This study shows 58\% females (M: F-1:1.38) and of 25-45 age groups are mostly affected from Upper G. I. problems. Commonest finding was Gastroduodenitis $(28.9 \%)$ and in $1.57 \%$ Carcinoma stomach was detected. Among other pathological findings, Antral gastritis (23.28\%), Active duodenal ulcer (8.95\%), chronic duodenal ulcer $(1.45 \%)$, Gastric ulcer $(1.05 \%)$ and normal findings in UGIE were in $4.86 \%$ cases.

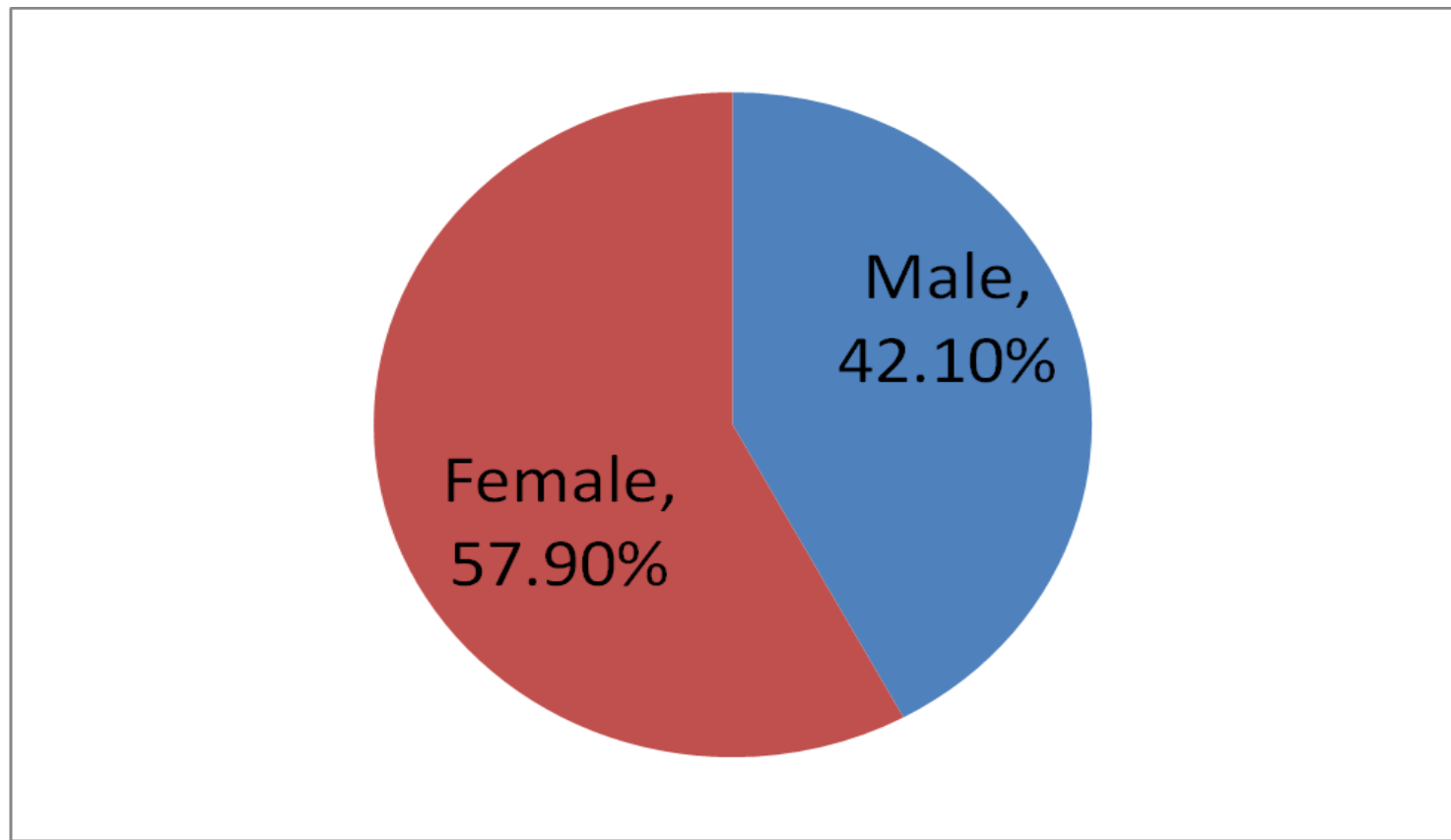

Figure 01: Sex distribution

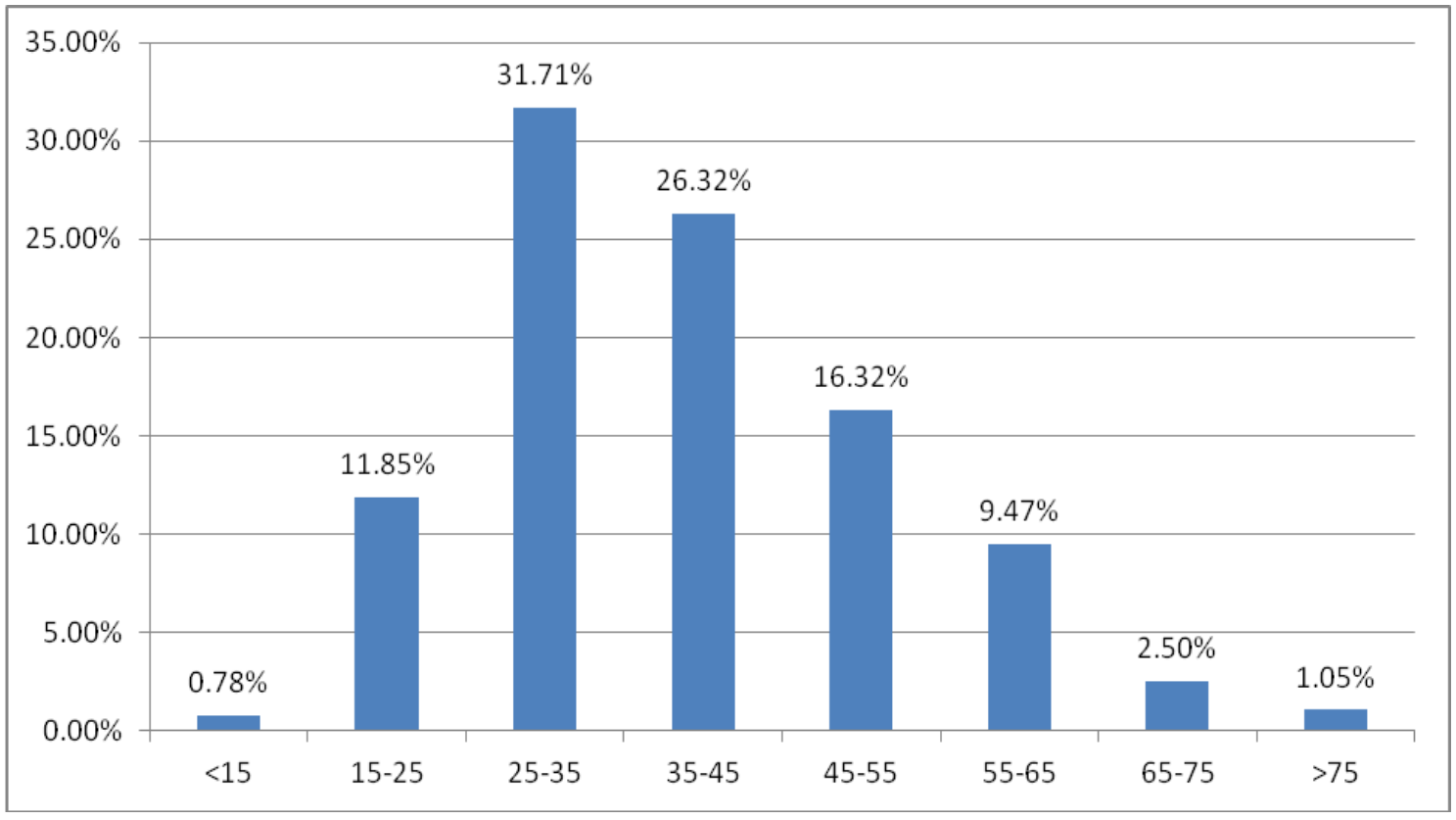

Figure 02: Age Distribution of cases 


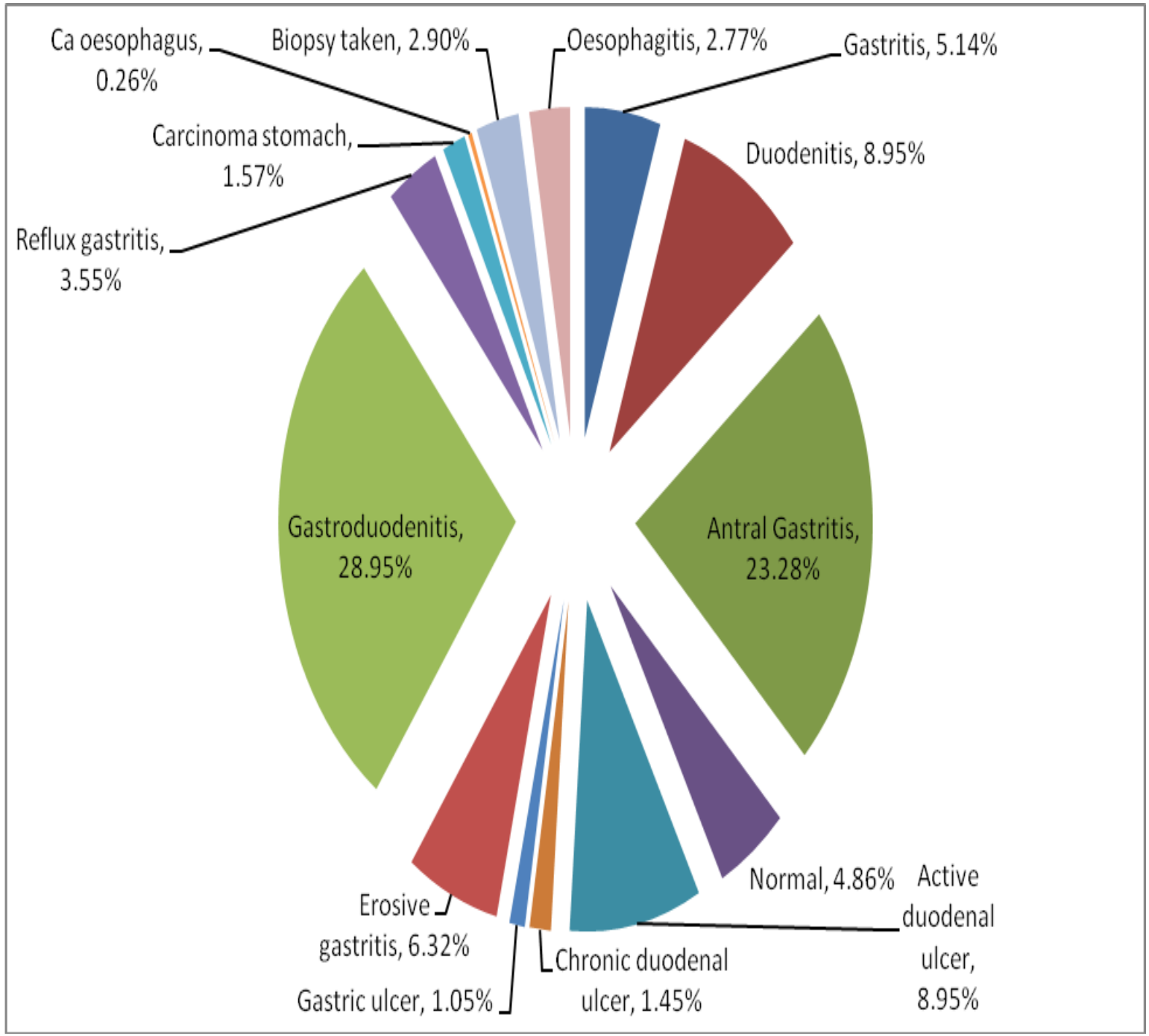

Figure 03: Specific Pathologies

\section{DISCUSSION}

Upper G.I. Endoscopy is a very safe procedure if performed by trained personnel it is very difficult to diagnose many conditions endoscopically like gastritis, duodenitis, erosive gastritis etc. but experience teaches all these and can be diagnosed. Erosion and Ulcer are the histological diagnosis. Endoscopically erosions are diagnosed where there are $>1$ lesion, less than $5 \mathrm{~mm}$ diameter and shallow with no signs of scarring. ${ }^{1}$

Oesophagitis normally follows acid reflux and is most apparent distally close to the mucosal junction. The earliest changes consist of mucosal congestion \& edema, which obscure the normal fine vascular pattern. Strictures from primary esophageal carcinoma are usually asymmetrical; there are areas of exuberant abnormal mucosa and raised edges to any ulcer. The classical chronic benign gastric ulcer is usually single and is most frequently seen on the lesser curvature at or above the angulus, it is typically symmetrical with smooth margins and a clean base. Features favoring malignancy include raised irregular margins, an irregular lumpy hemorrhagic base and mucosal abnormality surrounding the ulcer. ${ }^{2,3}$

Duodenal ulcers occur on the anterior and posterior walls of the bulb and are frequently multiple, when active they are surrounded by edema and acute congestion. Biopsy and cytology samples are not usually taken from bleeding lesions. Endoscopy should be repeated for this purpose after the acute phase-if the surgery has not been done. ${ }^{4,5}$

Problems of gastrointestinal function are usually not well diagnosed by endoscopy since motion or secretions of the gastrointestinal tract are not easily inspected by UGIE. Nonetheless, findings such as excess fluid or poor motion of gut during endoscopy can be suggestive of disorders of function. Irritable bowel syndrome and functional dyspepsia is not diagnosed with UGIE, but UGIE may be helpful in excluding other diseases that mimic these common disorders. ${ }^{6}$ 
Risk Factors for Complications during the Procedure are Age: 60 or older, Pregnancy, Obesity, Smoking, Malnutrition, Recent or chronic illness, Diabetes, Heart or lung problems, Bleeding disorders, Alcoholism, Use of certain medications, including blood pressure pills, muscle relaxants, tranquilizers, sleeping pills, insulin, steroids, sedatives, and hypnotic agents, Use of street drugs, including LSD, hallucinogens, marijuana, and cocaine. ${ }^{7}$

\section{ACKNOWLEDGEMENT}

I would like to thank Dr. Basanta Kumar Tamrakar in helping to do the procedure, Dr. Duk Bahadur chhetri for histopathological examination and Dr. Prajwal Ghimire for computer works.

\section{REFERENCES}

[1]. De Vault KR, Castell DO. Guidelines for the diagnosis and treatment of gastrooesophageal reflux, practice and parameters committee of the American college of Gastroenterology. Arch Intern Med 1995, 155: 2165-2173.

[2]. Spechler SJ, Zeroogian JM, Antonioli DA etal. Prevalence of metaplasia at the gastro-oesophageal junction. Lancet 1994, 344: 1533-1536.

[3]. Peter B. Cotton, Christopher B. Williams. Practical Gastrointestinal Endoscopy: Second edition.

[4]. Bailey \& De Vault KR, Castell DO. Guidelines for the diagnosis and treatment of gastro esophageal reflux disease, practice and parameters committee of Love's Short practice of surgery. 23rd Edition 2000.

[5]. A. Cuschien, G.R. Giles, A.R. Moosa, Essential Surgical Practice, third edition 1995

[6]. www.wikipedia.org/ esophagogastroduodenoscopy

[7]. Rosalyn Carson-DeWitt. Upper GI Endoscopy (Upper Gastrointestinal Endoscopy, Esophagogastroduodenoscopy [EGD]) ; Nucleus Medical Art, Inc. 2008 